\title{
The application of bronchostomy to the experimental study of transplanted pulmonary tissue
}

\author{
WILLIAM HEDLEY JONES \\ From the Royal Northern Hospital and the Royal Free Hospital Group, London
}

The progress of a grafted canine kidney introduced into a bilaterally nephrectomized dog may be followed by the estimation of the blood urea or the serum creatinine level at intervals. Alternatively, urine from the grafted kidney may be analysed and the secretory function of the kidney inferred from the concentrations of the chemical constituents within it (Calne, 1961). The fate of a canine liver homograft may similarly be followed by observation of the biliary secretion collected from a cannula introduced into the gall bladder after ligation of the common bile duct (Goodrich, Welch, Nelson, Beecher, and Welch, 1956). In a hepatestomized dog, the function of an orthotopically transplanted liver homograft may be determined by the periodical investigation of the serum bilirubin or alkaline phosphatase level (Starzl, Kaupp, Brock, and Linman, 1961).

The functional progress of the grafted lung is less easily assessable than that of the kidney or the liver because (1) bilateral pneumonectomy with the implantation of a pulmonary graft is a major operation, and no record of survival of this as a single procedure has been found; and (2) denervation of all functional pulmonary tissue is accompanied by abolition of the Hering-Breuer reflex, which leads to slow, deep respiration incompatible with prolonged satisfactory existence. Though contralateral pneumonectomy may be delayed as long as six months after excision and reimplantation of a lung, the procedure has not on any occasion resulted in long-term survival of the animal (Alican and Hardy, 1963).

The observations of the Russian surgeon Demikhov, in 1947, translated into English by Basil Haigh (1962), show that he was probably the first to transfer pulmonary homografts in dogs. Later, the study of lung transfer was abandoned by him in favour of the transplantation of the heart and lungs together as a unit, mainly due to the difficulties of the functional assessment of pulmonary grafts. Although intubation techniques have been used for the investigation of pulmonary function, these are usually used during general anaesthesia so that frequent determinations are hazardous and the behaviour of the graft is studied under artificial conditions.

In the present study, the airway of the left diaphragmatic lobe has been isolated from that of the undisturbed right lung of each animal, so that expired air leaving the left diaphragmatic lobe may be directly collected and analysed.

\section{MATERIAL AND METHODS}

Attention was confined to the diaphragmatic lobe of the left lung of the dog, which corresponds to the lower lobe of the human left lung, and its fate was observed in 19 dogs. Left apico-cardiac lobectomy was performed in each animal (Fig. 1) and this was followed by one of four further procedures, the nature of which determined the group to which each animal was assigned.

GROUP 1 The only animal in this category was donated a left diaphragmatic lobar homograft from another dog. The graft was substituted for the left diaphragmatic lobe of the recipient animal, and its function was restored after end-to-end anastomosis of the stumps of the lobar bronchus, pulmonary artery, and vein respectively. Isolation of the airway of the grafted lobe was effected by endobronchial intubation of the left main bronchus with a balloon catheter which had been introduced through a tracheostomy (Fig. 2).

GROUP 2 Anatomical isolation of the natural airway of the left diaphragmatic lobe was established by transposition of the left main bronchus and its continuation distally as the diaphragmatic lobar bronchus, so that its open end emerged through the dorsal part of the thoracotomy wound as a bronchostomy (Fig. 3). Diversion of the airway alone was carried out in one animal; the other was subjected to excision and reimplantation of its left diaphragmatic lobe.

GROUP 3 A tube was used to replace the normal extrapulmonary bronchial passages and was led outwards through the dorsal end of the chest wound (Fig. 4). The airway was fashioned in this way in three dogs; one was subjected to bronchostomy only, the second to 


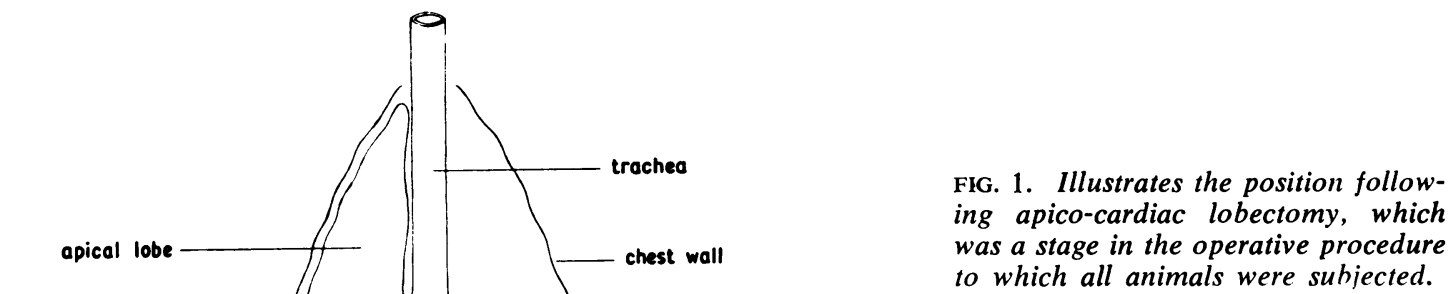

FIG. 2. Demonstrates the result of surgical intervention in the animal of group 1. End-to-end anastomosis of the left diaphragmatic lobar bronchus was accompanied by intubation of the left main bronchus using a Foley catheter which had been introduced through a tracheostomy.

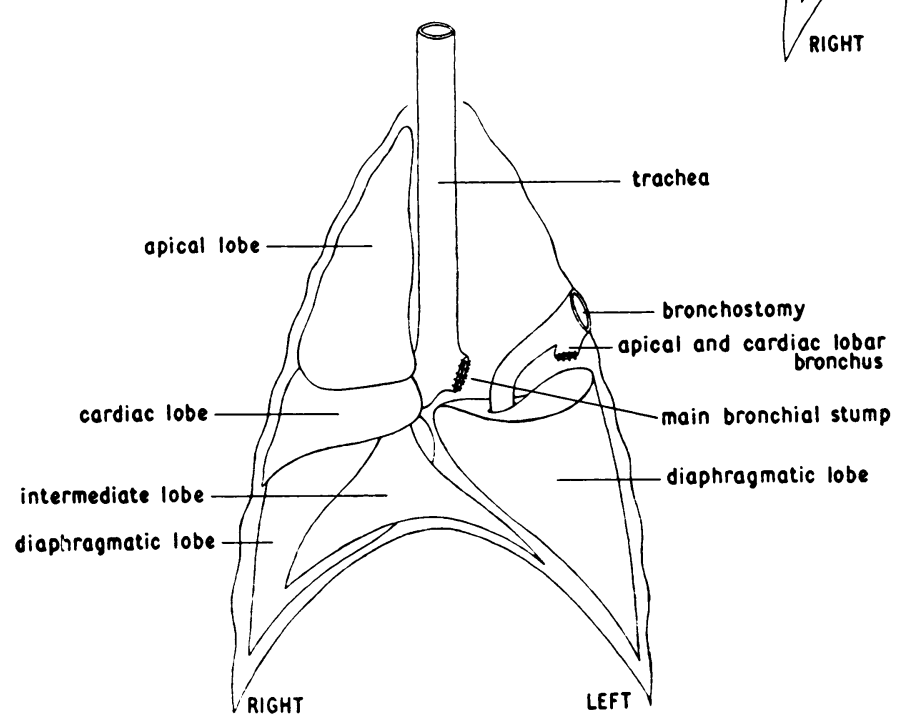

FIG. 3. Depicts the pattern in animals of group 2. The left main bronchial stump has been closed, and the course of the left main bronchus and its continuation as the diaphragmatic lobar bronchus has been diverted so that it communicates with the surface of the chest as a bronchostomy. 
FIG. 4. Shows how the left main bronchus and its continuation distally to the diaphragmatic lobe has been replaced by a tube which has been brought out through the chest wall in all animals of group 3.
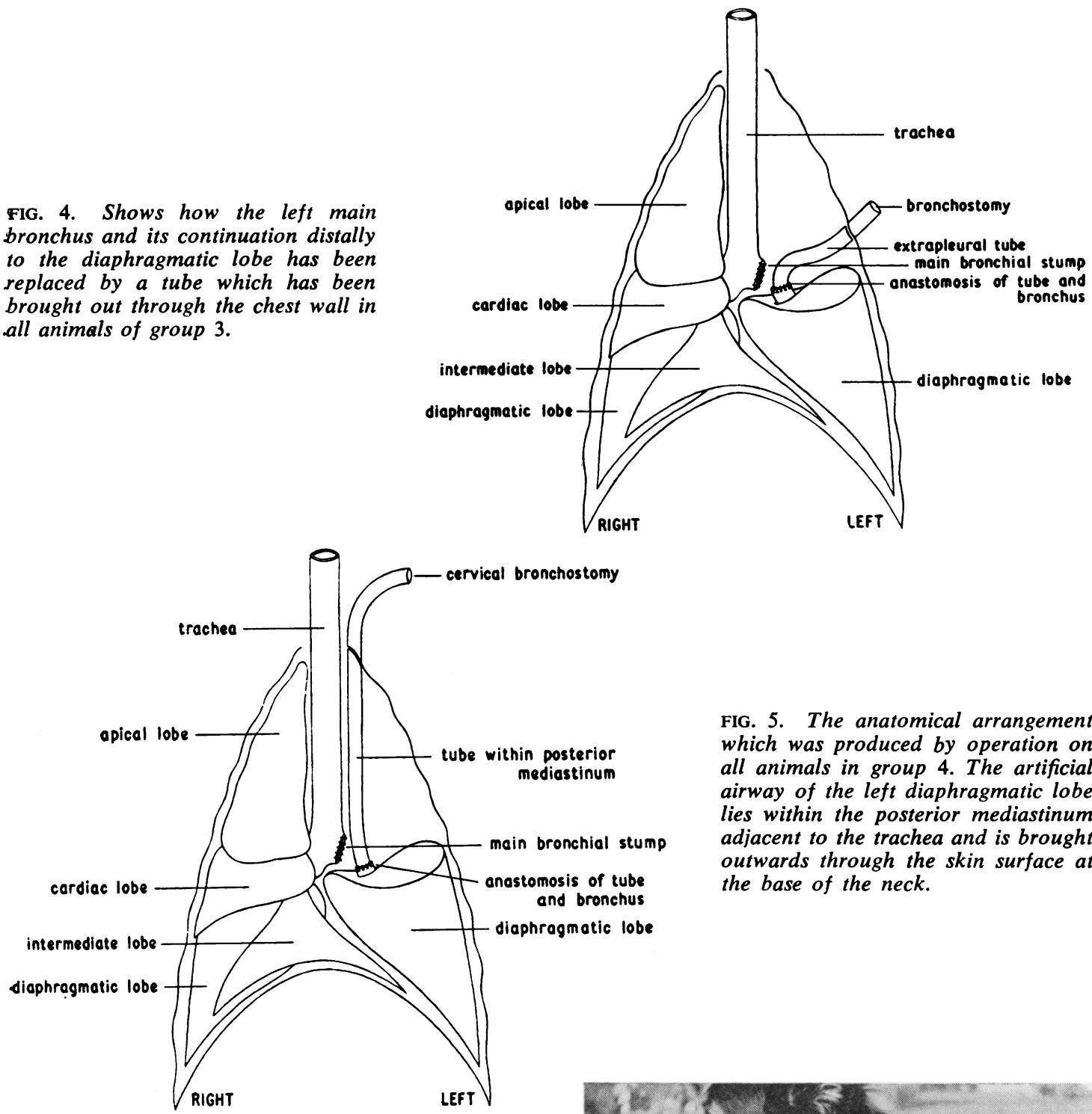

FIG. 5. The anatomical arrangement which was produced by operation on all animals in group 4. The artificial airway of the left diaphragmatic lobe lies within the posterior mediastinum adjacent to the trachea and is brought outwards through the skin surface at the base of the neck.

FIG. 6. An animal of group 4 on the second day after left pneumonectomy and receipt of a left diaphragmatic lobar homograft. The airway of the grafted lobe is seen emerging through the skin surface at the base of the left side of the neck.

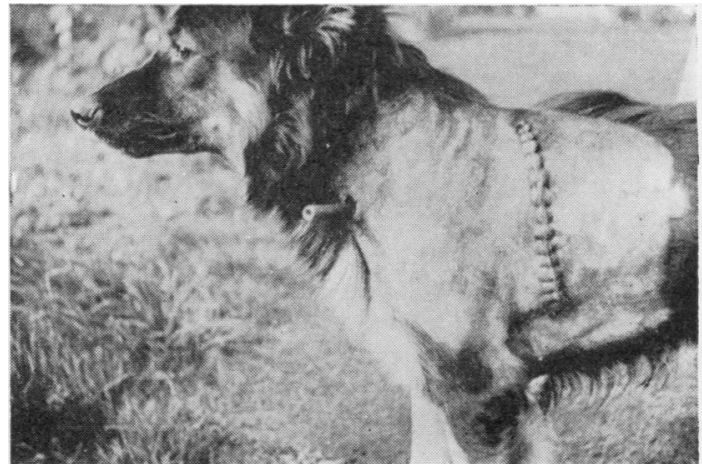


excision and reimplantation of its left diaphragmatic lobe, and the third was donated a left diaphragmatic lobar homograft from another dog.

GROUP 4 In principle, the technique used was comparable with that used in animals of group 3, except that the course followed by the artificial conduit was different, being mediastinal alongside the trachea and perforating the surface at the base of the left side of the neck (Figs 5 and 6). There were 13 animals in this group. One of these was given a cervical bronchostomy only, without division of the lobar vessels. In five dogs, the diaphragmatic lobe was excised and reimplanted, and seven were the recipients of diaphragmatic lobar homografts.

Soon after closure of the chest, and at convenient intervals thereafter, the ventilation of the left diaphragmatic lobe was measured using a Wright respirometer (Fig. 7) and the oxygen saturation of samples of expiratory gas was determined using a D.C.L. 101 Servomex oxygen analyser.

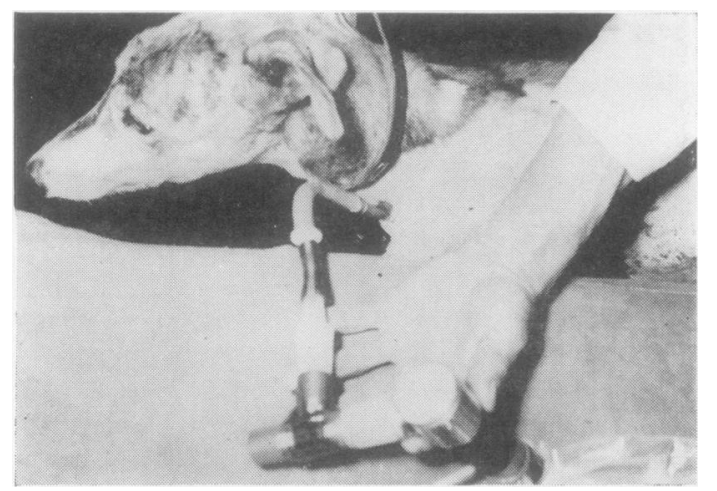

FIG. 7. An animal of group 4 standing patiently while expiratory gas is exhaled through a Wright respirometer and collected in a plastic bag of low diffusion permeability.

RESULTS

The results are considered in relation to each of the four groups of animals in the series.

GROUP 1 From the time of performance of tracheostomy initial ventilation of the right lung was unsatisfactory because of a leak of gases from the trachea at the tracheostomy site, but at the end of the operation the condition of the dog was satisfactory. Its breathing was not laboured and the tongue was pink and warm. The respiratory rate was 42 excursions/minute and the expired volume from the transplanted lobe was on average $1.4 \mathrm{l} . /$ minute.
On the following morning the dog, though $\frac{}{0}$ conscious, was dyspnoeic and the tongue was blue. It was taking water but dragged itself around, $\overparen{\nabla}$ unable to walk. Movement of the right chest was poor and breath sounds were diminished. Movement of the left chest was better than that of the $\vec{O}$ right, and breath sounds were easily audible. An $\overrightarrow{\vec{H}}$ inspiratory rhonchus was added to the sounds ${ }_{\sigma}^{\omega}$ heard over the left chest. The respiratory rate was $50 /$ minute and the volume of the expired air leaving the graft was $1.6 \mathrm{l} . /$ minute. The condition of the dog worsened later in the day; it showed signs of distress and gasped for breath. 앙 There was good excursion of respiratory gases through the Foley catheter, but the volume of the expired air was not measured. Resuscitation failed $\bar{c}$ and the dog died during the night.

Post-mortem examination revealed collapse of $\overrightarrow{\mathbb{D}}$ the right lung with much bronchial secretion $\frac{3}{0}$ within the trachea and throughout the bronchial $\stackrel{\mathbb{Q}}{-}$ passages of the right side of the chest. The left $\vec{\bullet}$ diaphragmatic homograft was much as it had been at the time of transfer, with no significant secretion within its airway. Though there were fragments of antemortem thrombus attached to the pulmonary arterial and venous anastomoses, these were small. Death was attributed to pul- $\frac{\circ}{\nabla}$ monary failure due to massive collapse of the right lung.

GROUP 2 The animal subjected to bronchostomy alone without disturbance of the pulmonary lobar: artery or vein survived until intentionally overdosed with pentobarbitone sodium on the sixth 음 day after operation. It was well for about two days after operation, though there was minimal $\frac{0}{3}$ excursion of respiratory gases through the bronchostomy stoma. Breath sounds over the lefto chest were poor during this period. Its temperature was raised on the third day after operation $\frac{\text { o }}{7}$ and food was refused. Coughing developed $>$ and serous fluid was produced from the stoma. The fluid became seropurulent and the condition $\bar{N}$ of the dog worsened. A bronchopleural fistula was ${ }^{\text {G }}$ suspected, and on bronchoscopy through the $N$ stoma on the sixth day after operation rupture $\mathbb{W}^{N}$ of the bronchus was demonstrated; the animalo was killed on the same day. Much seropurulente fluid was found in the pleural cavity; the $\frac{\bar{D}}{\mathscr{C}}$ bronchus was necrotic and had parted from the lobe.

The second animal in which excision and reimplantation of the left diaphragmatic lobe had $\stackrel{\mathbb{Q}}{\Omega}$ been carried out died on the third day after $\overrightarrow{\mathbb{D}}$ operation. On the day after grafting its generalo condition was satisfactory. It walked around, 
lapped water, and took some food. Excursion of gases through the stoma was, however, poor. It maintained its general improvement for another day but function of the reimplanted lobe was clearly no better. Next day it was rather less well; it lay around most of the time and refused food. Deterioration was rapid, culminating in death during the night. At necropsy the grafted lobe was oedematous and haemorrhagic, and the bronchus was white and structureless, suggesting necrosis. There was occlusion of the lumen of the pulmonary lobar artery, mainly by white clot, with similar occlusion of the pulmonary lobar vein.

GROUP 3 The dog which had been given a bronchostomy, where the diaphragmatic lobar vessels had not been disturbed, was sacrificed on the ninth day after operation. During its survival period it behaved normally, appearing well, taking food, and walking about. For the first three days after operation excursion of gases to and from the diaphragmatic lobe was good; subsequently the ventilation deteriorated and accumulation of sticky mucoid secretion became apparent in the tube. Aspiration of the mucus improved ventilation, but over a few days it became extremely difficult to clear the secretion from the tube and the ventilation gradually deteriorated until it ceased. Because of failure to re-establish ventilation the dog was killed with an overdose of pentobarbitone sodium on the ninth day. Exploration of the left side of the chest showed that, although the junction of the tube with the bronchial stump was intact, the lumina of the tube and bronchial passages were occluded by viscid secretion. There was no obvious intrapleural sepsis and the pleural cavity contained only a small amount of free fluid.

The second dog, subjected to excision and reimplantation of the diaphragmatic lobe, suffered cardiac arrest within a few minutes of re-establishment of function of the lobe after completion of the arterial anastomosis. This occurred as a result of accidental interruption of the oxygen supply. The heart beat was, however, restored by manual cardiac massage, and thereafter the heart continued to beat without further stimulation. During the four days of post-operative survival this animal displayed decerebrate rigidity. Good aeration of the reimplanted lobe was observed for two days after the operation, but on the third day it became poor and was interrupted by the ejection of small amounts of thin, sero-sanguineous discharge from the tube. On the last day of survival there was no air excursion through the tube, but increasing amounts of sero-sanguineous fluid were e;ected from it; later the dog died. Post-mortem examination showed necrosis of the lobar bronchial stump with a bronchopleural fistula. The resilience of the tube, which described a sigmoid course, had led to strain at the anastomosis of the tube and bronchus with subsequent bronchial necrosis and disruption.

The third dog, which was donated a lobar homograft, appeared to be well during its survival period, but because there was no ventilation of the transplanted lobe the animal was killed on the second day after operation. Ventilation of the grafted lobe was extremely good immediately after the operation, and though it was less marked on the day after operation, aspiration of gas and blood-stained fluid from the pleural cavity led to improvement. On the second day after operation no ventilation of the transplanted lobe was observed despite further aspiration of the contents of the pleural cavity. Exploration of the left side of the chest after death showed that the junction of the tube and the bronchus was sound and there was no obstruction of the airway. The transplanted lobe was readily inflated by positive pressure through the tube and no leak along the airway was detected. Both arterial and venous anastomoses were free of thrombus.

GROUP 4 Of the 13 dogs studied in this group, one was subjected to isolation of the airway only. This animal remained well for more than three months after operation. At the end of the operation the expired volume from the diaphragmatic lobe was $0.4 \mathrm{l} . / \mathrm{min}$., but on the following day the volume was unrecordable with the Wright respirometer. Subsequently there was no improvement. Ejection of a thin, purulent fluid from the opening of the tube was noticed on the third day after operation. This purulent discharge persisted throughout the survival period even though the general condition of the dog was extremely good.

Of the five dogs subjected to excision and reimplantation of their lobes, four dogs lived for less than a week and the fifth for 57 days. One dog died on the third day after operation, never regaining consciousness after anaesthesia; necropsy on this dog was not possible. Of the two dogs that died on the fourth day after operation, one developed a bronchopleural fistula, the other died of pulmonary venous thrombosis. The dog that survived to the sixth day was also found to have pulmonary venous thrombosis, and it was thought that its sudden death resulted from an acute systemic embolic episode. Excursion of 
respiratory gases through the cervical bronchostomy tube was demonstrated throughout the survival period in all four animals that survived less than a week.

The dog that survived for 57 days remained well throughout this period. It took its food for most of the time and behaved normally. For experimentation purposes limitations were imposed by the small size of the dog which weighed $14 \mathrm{~kg}$. Consequently the expiratory volume was never sufficiently large to be recorded by the Wright respirometer, which is, of course, designed for human clinical practice. Furthermore, collection of expired air for sampling was difficult as the volume was small. As the total 'dead space' of the airway and valve was relatively great there was contamination of the expired air with atmospheric air, and at no time was the oxygen saturation ever less than $20 \%$.

The greatest interest in this case centred on the functional pattern of the reimplanted lung. For the first three post-operative days gaseous exchange was reflected by flow and counterflow at the bronchostomy stoma. This was confirmed objectively by the visual movement of a thin piece of paper applied to the stoma. By the fourth day the amplitude of flow movements had decreased to zero, and it was inferred on this basis that there was no detectable functioning of the graft. During these four days the temperature was never higher than $102 \cdot 6^{\circ} \mathrm{F}$. (the normal for dogs being $101 \cdot 2^{\circ} \mathrm{F}$.), but during the fifth to the eighth day the temperature rose to $103.6^{\circ} \mathrm{F}$, gradually returning to normal limits on the tenth day. On this day exchange of respiratory gases was observed at the stoma and persisted for six days. Unfortunately, the protruding portion of the tube was bitten off by the dog, and this resulted in the withdrawal of the stoma beneath the skin surface, thereby obstructing it. Further procedures were performed in an attempt to maintain the patency of the airway and restore the function of the reimplanted lung, but these were without success.

Three of the seven dogs given homografts were provided with lung lobes which had been removed from dogs, stored in the refrigerator at $4^{\circ} \mathrm{C}$. for three to five hours, and then transferred to the recipient animals. Each of these dogs succumbed within 24 hours of completion of the operation, one within minutes of closure of the chest. In all these dogs copious amounts of colourless, thin fluid issued forth from the cervical bronchostomy stoma soon after circulation in the graft had been restored, and this persisted in each case until the death of the animal.
Of the four remaining dogs that were donated homografts, three were given tubocurarine chlor- $\frac{\bar{D}}{\bar{D}}$ ide. This abolished the spontaneous movement of $\mathbb{Q}$ the thoracic cage and considerably eased anastomosis of the pulmonary arterial and venous

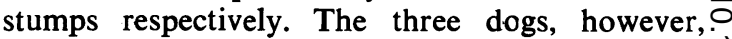
died within 24 hours of completion of the pro- $\vec{\omega}$ cedure. After the operation injection of neostig- $\omega$ mine intramuscularly, and in excess of the calcu- $\vec{x}$ lated neutralization dose, was administered with atropine to the three animals. Muscular power ir was enhanced in all of them and was associated with improved respiratory movement. One of the or animals was, however, relatively resistant to을 neutralization with neostigmine and atropine, and on three occasions injection of this drug wases followed by temporary benefit only. On each occasion this dog was able to raise its head and $\overrightarrow{\mathbb{D}}$ lap fluids but was unable to support itself in the pronograde position. These effects were always $\stackrel{\Phi}{?}$ only temporary and the state of flaccid paralysis $\vec{\theta}$ was then resumed.

The remaining dog (Fig. 6), which was donated a pulmonary homograft, survived for nine days, when it was killed by an overdose of pentobarbitone sodium for further investigation. The graft was directly transferred from a greyhound to this $ळ$ mongrel dog, and anastomoses were carried out $\stackrel{\varrho}{\Rightarrow}$ without the use of tubocurarine. The junction of $\frac{O}{3}$ the bronchial stump with the armoured tube was covered with a pericardial flap. During the survival period this dog was well; it took food and was extremely active. Its temperature rose steadily from the normal $\left(101 \cdot 2^{\circ} \mathrm{F}\right.$.) to $104.8^{\circ} \mathrm{F}$. on the fifth day after operation and fluctuated between this and $103.6^{\circ} \mathrm{F}$. until it was killed. On the day after operation its respiration rate was 42 excursions/min. and the expired volume from the 3 grafted lobe was $3.01 . / \mathrm{min}$. The oxygen satur- 0 ation of the expired air was $19 \cdot 6 \%$. The ventilation was reduced to $0.81 . / \mathrm{min}$. on the secondo day, and analysis of samples of expired air on this day showed that the oxygen saturation wasc $19.1 \%$. The volume of the expired air was in- N sufficient for recording with the Wright respirometer on the third day after operation, but ${ }_{\sigma}^{\omega}$ samples taken from a bag of exhaled air showed the oxygen saturation to be $20.4 \%$. From then onwards to the time when the dog was killed, the expiratory volume was minimal, and it was 0 impossible to obtain samples for oxygen analysis. 0 On the sixth day mucopurulent secretion was seen within the tube, and this persisted throughout the remainder of the survival period. Necropsyo showed oedema of the lobe with mottling of its 
surface. Histologically, the appearances were consistent with total infarction with secondary infection, indicative of the end result of immunological rejection.

\section{DISCUSSION}

The study was designed with the purpose of providing a simple, safe way of determining the function of a pulmonary graft under normal conditions. Other workers (Nigro, Reimann, Mock, Fry, Benfield, and Adams, 1963) have carried out functional studies on dogs which had been subjected to pneumonectomy and pulmonary reimplantation using intubation techniques to isolate the respiratory gases of the graft. These investigations have, however, necessitated general anaesthesia, and recordings at frequent intervals are therefore not easily available.

Whereas reimplanted pulmonary tissue will survive indefinitely and regain almost normal function, pulmonary homografts are uniformly found to become necrotic by the sixth day after operation (Blumenstock, Collins, Hechtman, Thomas, and Ferrebee, 1962). In this respect, pulmonary homografts differ from renal homografts, which function, according to Dempster (1953), for a period of up to 18 days before obvious deterioration occurs. It was therefore considered that, in the experimental study of pulmonary grafts, as sensitive a means as possible was necessary to reflect the altered function of the transplant. The technique was therefore developed (Jones-in press) whereby expiratory gas could be collected without disturbance of the animal (Fig. 7) and samples analysed both qualitatively and quantitatively.

Initially, the airway of the grafted lobe was cannulated with a cuffed catheter, which traversed the trachea, and was brought to the surface at the neck through a tracheostomy. This avoided disturbance of the normal morphological pattern of the bronchial tree. The presence of the catheter within the trachea was not well tolerated, however, and its irritation of the tracheal wall led to the production of excessive bronchial secretion. This produced massive collapse of the otherwise undisturbed right lung, and early death of the animal ensued.

Technical success was achieved by surgical transposition of the airway of the graft with isolation of that of the undisturbed right lung. Owing to the limited length of the extrapulmonary bronchial passages, however, this led to tension within the left main bronchus and its continuation as the diaphragmatic lobar bronchus. Necrosis of the bronchus followed this procedure with formation of a bronchopleural fistula. An additional complication in one animal was thrombosis of the pulmonary artery which had been 'hitched up' in an endeavour to obtain satisfactory bronchocutaneous anastomosis.

Insufficient length of the normal bronchial passages necessitated the insertion of a prosthesis for conduction of the respiratory gases to the skin surface. With the chest already open, it seemed expedient to select a route that utilized the incision and necessitated little further surgical intervention. The selected pathway was unavoidably tortuous, however, and the resilience of the armoured tube led to pressure necrosis of the bronchial wall. The use of a linear course for the tube would avoid this complication, and so the technique used in animals of group 4 was adopted.

In group 4 , the artificial conduit approximated to the course of the normal airway. Short-term results were encouraging, but disruption at the junction of the tube and bronchus led to suppuration around the tube and to bronchopleural fistula formation. The anastomosis of the tube to the bronchus was subsequently protected by envelopment with a pericardial flap. Improved results were observed after the use of this technique, particularly when it was supported by the systemic administration of a combination of antibiotics, usually tetracycline and chloramphenicol. With the introduction of tissue adhesives it was decided to test the efficacy of the Eastman monomer 910 in producing union between the bronchial stump of the graft and the tube. No major disadvantages were encountered and the junction remained sound in both animals tested. Blind intubation of the mediastinum was undertaken with hesitation in view of the potential hazards involved; but this manipulation was accompanied by no special complication.

The use of cadaveric homografts, temporarily stored in the refrigerator, proved unsuccessful in the present series of experiments, and this is presently attributable to the release of noxious substances by the doubtfully viable lung tissue into the systemic circulation. The pulmonary oedema was first noticed immediately after reestablishment of the circulation within the graft in each instance and persisted until the death of the dogs so treated.

Tubocurarine chloride provided ideal operating conditions, particularly at the most difficult stage when re-anastomosis of the pulmonary vessels 
was being carried out. The paralysis induced by this drug was, however, relatively resistant to treatment with neostigmine in combination with atropine in all three animals, particularly so in one of them. In view of this the use of tubocurarine chloride was discontinued in the present work.

Of the techniques used to date, that involving cervical bronchostomy, as in the experiments of group 4, has shown the most promise. By this method, reimplanted pulmonary lobes have been found to ventilate spontaneously and to allow gaseous exchange to occur within them. The spontaneous ventilation of a reimplanted lobe after grafting in one dog was interrupted by an episode of non-function presumed to have been due to pneumonitis, but ventilation was resumed on return of the temperature of the dog to within normal limits. In this instance, aeration of the graft was finally disturbed by the dog biting off the protruding portion of the bronchostomy tube, whereby the stoma was occluded. Homografts were shown to function satisfactorily for the first 48 hours after grafting, but this ceased concomitantly with rising temperatures. These features are the manifestations of the initiation of the foreign tissue rejection, resulting in necrosis of the graft.

The Wright respirometer was barely suitable for measurement of the expiratory volume in view of the relatively high inertia of the instrument at low flow rates. In the bigger dogs, where the pulmonary grafts were larger, the flow through the respirometer was measurable. In small dogs, greater accuracy was obtained by collecting expired air in a bag over a timed period. From this the volume of the expired air per unit time could be calculated.

The Ruben valve, too, revealed limitations, particularly for use in the smaller dogs, for the 'dead space' of the valve together with that of the airway was relatively large in comparison with the tidal volume of the graft. This caused contamination of the expiratory air with atmospheric air, with the result that oxygen saturation of the expired air was always greater than expected.

\section{SUMMARY}

A technique has been devised for the isolation of the airway of the diaphragmatic lobe of the canine left lung.

By this means technical isolation of the airway of grafted left diaphragmatic lobes has been satisfactorily obtained in a number of animals over limited survival periods.
In one animal, the ventilation of a reimplanted lobe was abolished during a period of pyrexia, reappearing spontaneously with persistence of this $\bar{\sigma}$ state on return of the temperature to within normal limits.

Ventilation of homografted lobes is abolished within 72 hours of operation, coincident with the systemic manifestation of the onset of rejection. and never occurs again.

This work was undertaken with financial support, or in the form of research grants, from the Royal Free or Hospital and the Medical Research Council, and Io am grateful for this. I am also indebted to Mr. George Qvist for his support throughout the investigation : to $\vec{c}$ Professor Charles B. B. Downman and Dr. J. R. D Hodges for their kindness in giving me experimental facilities at the Royal Free Hospital School of Medi- $\frac{D}{3}$ cine; to the President and Council of the Royal College of Surgeons of England for facilities at the Buckston Browne Surgical Research Farm ; to Profes- $\vec{\bullet}$ sor David Slome for his encouragement; to Dr. T.S Hilary Howells for advice on problems in anaesthesia and for his enthusiasm; to Professor Herbert Spencer for undertaking responsibility for the histological aspect of the project; to Professor Don R. Arthur. $\bar{O}$ with whom I have discussed the scientific implications of the work, for his criticism of the manuscript; to $\varrho$ Miss Frances M. Ellis and the Photographic Depart- $\overrightarrow{\vec{A}}$ ment of the Royal Free Hospital for photographic $\frac{0}{3}$ assistance; and to many other persons, including technical staff. in particular Mr. Anthony R. H. Jowers, Miss Diane O'Grady, Mr. F. Watson, and Mr. Norman Thurlow. medical students, and nursing staff who spared much time to this project.

It is a pleasure to record my thanks to my wife $x$ Jane for the final presentation of the script.

\section{REFERENCES}

Alican, F., and Hardy, J. D. (1963). Lung reimplantation. Effect on respiratory pattern and function. J. Amer. med. Ass., 183, 849.

Blumenstock, D. A., Collins, J. A., Hechtman, H. B., Thomas, E. D. D and Ferrebee, J. W. (1962). Functioning homografts of the lung in dogs. Ann. N.Y. Acad. Sci., 99, 882.

Calne, R. Y. (1961). Inhibition of the rejection of renal homografts in $\mathbb{G}$ dogs by purine analogues. Plast. reconstr. Surg., 28, 445.

Demikhov, V.P. (1962). Experimental Transplantation of Vital Organs. N (Authorized translation from the Russian by Basil Haigh.) Consultants Bureau, New York.

Dempster, W. J. (1953). Kidney homotransplantation. Bit. J. Surg., 40, 447.

Goodrich, E. O., Welch, H. F., Nelson, J. A., Beecher, T. S., andeo Welch, C. S. (1956). Homotransplantation of the canine liver. Surgery, 39, 244.

Jones, W. Hedley (1966). The development of cervical bronchostomy $\stackrel{\mathscr{C}}{+}$ in relation to canine pulmonary transplantation. Brit.J. Surg. In the press.

Nigro, S. L., Reimann, A. F. Mock, L. F., Fry, W. A. Benfield, J. R.. and Adams, W. E. (1963). Dogs surviving with a reimplanted $\overparen{D}$ lung. Bronchospirometric and pulmonary artery pressure studies. J. Amer. med. Ass., 183, 854.

Starzl, T. E., Kaupp, H. A., Brock, D. R., and Linman, J. W. (1961) Q Studies on the rejection of the transplanted homologous dog liver. Surg. Gvnec. Obstet.. 112, 135. 